\title{
Four weeks of resistance training on muscular strength and ankle-brachial-index in osteoarthritis patients
}

\begin{abstract}
This study investigated the impact of a four-week resistance training program on muscular strength and ankle-brachial-index (ABI) in an osteoarthritis geriatric population. A randomized clinical control trial consisting of an intervention group performing resistance and cardio respiratory training while the control group performed cardio respiratory training. Lower extremity muscular strength and $\mathrm{AB}$ were measured in all subjects one week prior to and following the training period. The intervention group participated in resistance-training three days per week. Both groups participated in twenty minutes of cardiovascular activity two days per week during the four-week study. Fifteen subjects, 14 females and one male, were randomly assigned to an intervention group ( 6 females, 1 male, age $=67.5 \pm 5.1 \mathrm{yrs}$, height $=164.7 \pm 5.9 \mathrm{~cm}$, mass $=89.5 \pm 19.3 \mathrm{~kg}$ ) or control group $(6$ females, 0 male, age $=72.1 \pm 9.3 \mathrm{yrs}$.

Height $=164.7 \pm 5.4 \mathrm{~cm}$, mass $=81.6 \pm 23.4 \mathrm{~kg}$ ). No differences were observed between groups or across time for muscular strength variables and ABI. Trends towards significant correlation were observed with changes in hip abductor strength and $\mathrm{AB}$ $(\mathrm{p}=0.06)$, knee extensor strength and ABI $(\mathrm{p}=0.08)$. The current study suggests that a four-week resistance training program for the lower-body of osteoarthritis older adults does not result in significant improvements in muscular strength and ABI. However, there is a trend improving hip abductor strength and $\mathrm{ABI}$ alterations.
\end{abstract}

Keywords: Rehabilitation, Peripheral vascular disease, Osteoarthritis
Volume I Issue 2 - 2017

\author{
Michael Turner, Katrina Hall-Breece, Tricia \\ Hubbard-Turner \\ Department of Kinesiology Charlotte, University of North \\ Carolina at Charlotte, Charlotte, NC 28223, USA
}

Correspondence: Michael J.Turner, Department of Kinesiology, Laboratory of Systems Physiology, UNC Charlotte, Charlotte, NC 28223, USA Email miturner@uncc.edu

Received: September 30, 2016 | Published: March 22, 2017

\section{Introduction}

Approximately 46.4 million adults over the age of 65 years are living in the United States with diagnosed osteoarthritis (OA) and a significant amount of these individuals are physically inactive. ${ }^{1}$ According to the $\mathrm{CDC}$, individuals with hip and knee OA have a higher incidence of years living with disability compared to those with $\mathrm{OA}$ at other joint areas. ${ }^{2} \mathrm{OA}$ results in a decline in physical activity, functionality, cardiovascular function, quality of life, and an increase in joint pain..$^{3-5}$ The negative consequences associated with physical inactivity and OA necessitates the need for investigation into methods of prevention and treatment.

Improving muscular strength in patients with knee OA has been a priority of researchers and clinicians to prevent further disability. Strengthening of quadricep, hamstring, hip flexor, and hip abductor muscle groups has been reported to improve functionality and decrease risk for falls. ${ }^{4}$ Studies have also shown that a weak vastuslateralis and gluteus medius increase the risk for falls and decrease functionality. ${ }^{4,6,7}$ Decreases in muscular strength are negative consequences of aging, and losses in muscular strength appear to be magnified in patients with OA. These changes may also influence vascular function, which could explain the cardiovascular dysfunction experienced by patients with OA.

Ankle-brachial-index (ABI) is a measurement to assess peripheral artery risk as a result of the narrowing of the arteries in the legs and ankle. Recent research has reported high $\mathrm{ABI}$ values to coincide with decreased muscular strength. ${ }^{8}$ Determination of whether OA-related muscular weakness leads to impairment of lower body vasculature function has yet to be investigated. ${ }^{8,9}$

Numerous studies have researched resistance training (RT) in geriatric populations, ${ }^{4,6,7}$ but little is known regarding the influence of RT on muscular strength and ABI. ABI in particular has yet to be investigated as a result of physical training in an OA population. Importantly, ABI might be a useful tool to determine if lower body vasculature stenos are is present in OA populations because of their known extremity weakness. Further research is needed to understand if lower $\mathrm{ABI}$ is present in those with $\mathrm{OA}$, and whether changes in strength can influence $\mathrm{ABI}$ in OA populations. Therefore, the purpose of this study is to investigate the effects of a RT program on muscular strength and $\mathrm{ABI}$ in an OA population. We hypothesize that a RT program will increase muscular strength and improve $\mathrm{ABI}$.

\section{Methods}

\section{Participants}

Subjects were volunteers recruited from community senior centers. All subjects were diagnosed with knee OA prior to inclusion into the study. Individuals were excluded from the study if they demonstrated signs or symptoms that may contribute to heart disease. Those who have been diagnosed with peripheral artery disease, cardiovascular disease, history of a heart attack or stroke, stage II and/or stage III hypertension, cancer or history of cancer in affected legs, rheumatoid arthritis, polio or post-polio, moderate to severe dementia, or chronic neurological disorders that may have attributed to nerve damage in the lower extremity were also excluded from the study. Those who received a catheterization or saphenous vein graft were excluded from 
the study as well. Individuals were excluded if they possessed an ABI greater than 1.50 or if they have participated in resistance training for at least 4 weeks prior to enrolling in the study.

After initial pre-screening to ensure subjects met inclusion and exclusion criteria, participants were randomly assigned to one of two groups. Fourteen subjects, 13 female and one male, were randomly assigned to an intervention group ( 7 females, 1 male, age $=67.50 \pm 5.13 \mathrm{yrs}$, height $=164.69 \pm 5.88 \mathrm{~cm}$, mass $=89.47 \pm 19.29 \mathrm{~kg}$ ) or control group $(6$ females, age $=72.10 \pm 9.31 \mathrm{yrs}$, height $=164.69 \pm 5.39 \mathrm{~cm}$, mass $=81.55 \pm 23.42 \mathrm{~kg})$. Ten participants were African-American (4 controls and 6 interventions) and four participants were European Americans ( 2 controls and 2 interventions). All subjects provided written informed consent prior to participation in the study, and the protocol was approved by the Institutional Review Board (IRB) at The University of North Carolina Charlotte.

\section{Exercise training procedures}

The intervention group participated in resistance training three days per week during the 4 week program while the control group did not participate in any resistance training. Additionally, both groups participated in twenty minutes of cardio respiratory (CR) activity at a pre-intervention pace (mph or rpm based on mode of exercise) determined by the six-minute walk test. CR training intensities were kept constant throughout the study in both groups. All participants participated in CR activity on the treadmill or recumbent cycle ergo meter two days a week. The intervention group participated in RT three times per week for four weeks, in addition to the two CR training bouts per week. Participants in the intervention group performed CR activity on the same day of resistance training (RT), leaving one resistance-training day without CR activity. RT for the intervention group included five machine weight exercises (knee curl, knee extension, hip abduction, hip adduction and leg press), four ankle exercises (plantar flexion, dorsiflexion, inversion and eversion) with resistance bands, and three functional exercises (timed sit-to-stand, timed knee lifts on the floor, timed step ups on a flat bench). Two sets of eight to 15 repetitions were completed with all machine weights during exercise programming. If the last two or three repetitions were relatively easy, two repetitions were added to each set. If the repetitions exceeded 15, then the weight was increased and the repetitions were decreased to eight. Ten to twelve repetitions at each ankle joint motion were performed for ankle exercises; band intensity was determined during first session, but was adjusted due to strength increases. Functional exercise timed two-minute bouts remained the same in all individuals and the amount of lifts, step-ups, or stands were recorded.

\section{Testing procedures}

Before assignment to the intervention or control group, and after the 4 week study period, muscular strength and ankle-brachial-index (ABI) were measured in all subjects. Height, weight, and body-massindex were also recorded before and after the intervention period for both groups. A six-minute walk test was administered to determine treadmill or recumbent cycle intensity before the intervention period. All pre- and post-intervention assessments took place in the late morning or early afternoon.

Muscular Strength: Muscular strength was measured with a MicroFet 2 (HOGGAN Health, West Jordan, UT) handheld dynamometer. Isometric peak torque strength for knee extension, knee flexion, hip flexion, extension, and abduction, and ankle plantar and dorsi-flexion were measured as previously reported..$^{10,11}$ The subject performed 3 trials of each movement and were instructed to push as hard as possible against the dynamometer for 5 seconds. The mean of the three trials were recorded per kilogram of body weight.

Ankle-Brachial-Index: Ankle-brachial-index (ABI) was used to determine the degree of stenos is in the lower limb vasculature. A sphygmomanometer was used to measure systolic blood pressure in the left and right upper arm and left and right lower leg. Blood pressures in each limb were measured twice for a total of eight blood pressure readings. The average systolic blood pressure for all arm measurements and leg measurements was recorded. ABI in this study was determined by using the lower ankle pressure (LAP) method; the lower ankle systolic ankle pressure was used as the numerator in this study. ${ }^{9}$ The ankle pressure was then divided by the average of the systolic brachial arm pressures. If the ABI calculation was less than 0.90 , then the subject was categorized as positive for insufficient circulation due to stenos is in lower limbs. If the subject received a score higher than 0.90 and lower than 1.50 , then $\mathrm{ABI}$ was considered to be negative for insufficient circulation and in normal range. ${ }^{8,9}$ If the $\mathrm{ABI}$ score was over 1.50 , the subject was considered to have poorly compressible legs and was excluded from the study.

Six-Minute Walk Test: The six-minute walk test was used to determine treadmill and recumbent cycle exercise intensity for both groups. The test was performed around a gym-marked floor that measured 270 feet in length. The participant was encouraged to walk as many laps as possible in six minutes at an intensity of thirteen (somewhat hard) on the rating of perceived exertion scale (Borg scale; 6-20 point). After the conclusion of the test, the laps walked were recorded, in addition to excess feet and inches, as an indication of CR fitness. This information was used to determine the treadmill or recumbent cycle exercise intensity for both groups. ${ }^{12}$

\section{Statistical analysis}

Means and standard deviations were computed for each dependent variable by group and time period. A $2 \times 2$ design MANOVA with repeated measures was used to examine muscular strength, and ABI, changes between groups (Control, Intervention) and across time (Pre, Post). Additionally, Pearson-product moment correlations were used to determine the relationship between muscular strength and ABI. The level of significance was set $a$ priori at $\mathrm{p}<0.05$ for all analyses.

\section{Results}

Means and standard deviations for the descriptive characteristics of the groups are presented in Table 1. No differences were found between the groups for age $(p=0.30)$, height $(p=0.95)$, pre-weight $(\mathrm{p}=0.51)$, and pre-BMI $(\mathrm{p}=0.45)$, weight did not change across the study period $(\mathrm{p}=0.97)$ nor was there an interaction of group by time $(\mathrm{p}=0.77)$. The performance of the six-minute walk test was not different between groups prior to the initiation of the study $(\mathrm{p}=0.84)$. There was no significant change in six-minute walk test performance by the end of the study period $(\mathrm{p}=0.12)$. Also, there was no interaction effect for the six-minute walk test $(\mathrm{p}=0.14)$.

No significant differences were observed between groups or across time for muscular strength (Table 2). No interaction effects were found for any muscular strength measurements, except hip abduction. There was a trend towards a significant interaction for hip abduction $(p=0.06)$, with the intervention group demonstrating stronger hip abduction from pre-testing to post-testing measurements compared to the control group. Ankle-brachial-index did not exhibit a statistical significance between groups or across time (Table 3). Lastly, no group by time interaction was observed with ankle-brachial-index. 
Table I Mean $( \pm S D)$ Descriptive Characteristics for Control and Intervention Groups

\begin{tabular}{lll}
\hline & Control & Intervention \\
\hline Age $(\mathrm{yr})$ & $67.5 \pm 5.1$ & $72.1 \pm 9.3$ \\
Height $(\mathrm{cm})$ & $164.7 \pm 5.9$ & $164.7 \pm 5.4$ \\
Weight $(\mathbf{k g})$ & & \\
Pre & $196.8 \pm 42.4$ & $179.4 \pm 51.5$ \\
Post & $197.4 \pm 45.2$ & $180.4 \pm 52.0$
\end{tabular}

Six-min walk (m)

$\begin{array}{lll}\text { Pre } & 457.3 \pm 67.5 & 464.1 \pm 56.2 \\ \text { Post } & 433.1 \pm 95.7 & 472.0 \pm 44.4\end{array}$

Table 2 Muscular Strength for Control and Intervention Groups (Mean \pm SD)

\begin{tabular}{lllll}
\hline Strength Control & Intervention & $\begin{array}{l}\text { Group } \\
\text { (p) }\end{array}$ & $\begin{array}{l}\text { Time } \\
\text { (p) }\end{array}$ & $\begin{array}{l}\text { Group } \\
\text { x Time } \\
\text { (p) }\end{array}$ \\
\hline
\end{tabular}

\section{Plantar Flexion(N/kg)}

$\begin{array}{llllll}\text { Pre } & 0.163 \pm 0.043 & 0.217 \pm 0.071 & 0.26 & 0.27 & 0.23 \\ \text { Post } & 0.179 \pm 0.081 & 0.200 \pm 0.052 & & & \end{array}$

\section{Dorsi-flexion (N/kg)}

$\begin{array}{llllll}\text { Pre } & 0.174 \pm 0.06 \mathrm{I} & 0.228 \pm 0.096 & 0.37 & 0.33 & 0.4 \\ \text { Post } & 0.200 \pm 0.1 \mathrm{II} & 0.225 \pm 0.064 & & & \end{array}$

\section{Knee Extension(N/kg)}

Pre $0.205 \pm 0.076$

$0.27 I \pm 0.117$

0.24

0.74

0.57

Post $0.204 \pm 0.068 \quad 0.252 \pm 0.087$

Knee Flexion (N/kg)

Pre $0.127 \pm 0.051$

$0.159 \pm 0.037$ 0.08

Post $0.113 \pm 0.044$

$0.165 \pm 0.043$

Hip Extension (N/kg)

Pre

$0.140 \pm 0.035$

$0.174 \pm 0.042$

0.09

0.26

0.18

Post $0.13 \mid \pm 0.055$

$0.189 \pm 0.058$

Hip Flexion (N/kg)

Pre $0.154 \pm 0.064$

$0.221 \pm 0.078$

0.25

Post $0.177 \pm 0.073 \quad 0.225 \pm 0.046$

\section{Hip Abduction (N/kg)}

Pre $0.138 \pm 0.042$

$0.181 \pm 0.079$

0.09

0.18

0.06

Post

$0.128 \pm 0.048 \quad 0.237 \pm 0.115$

No significant relationships were observed between changes in each strength measurement and ABI ( $>>0.05)$ (Table 4), except hip abduction strength and knee extension strength with ABI. Change in hip abductor strength and change in ankle-brachial-index exhibited a negative relationship for all groups $\left(\mathrm{r}^{2}=0.3311 ; \mathrm{p}=0.03\right)$ (Figure 1). Hip abductor strength change and ankle-brachial-index change exhibited no relationship with the control group $\left(\mathrm{r}^{2}=0.0025 ; \mathrm{p}=0.96\right)$ and exhibited a trend towards a negative relationship with the intervention group $\left(\mathrm{r}^{2}=0.4171 ; \mathrm{p}=0.08\right)$. Change in knee extensor strength and change in ankle-brachial-index exhibited a negative relationship for all groups $\left(r^{2}=0.3064 ; p=0.04\right)$ (Figure 2$)$. Knee extensor strength change and ankle-brachial-index change exhibited no relationship with the control group $\left(\mathrm{r}^{2}=0.4032, \mathrm{p}=0.18\right)$ and demonstrated a trend towards a negative relationship in the intervention group $\left(\mathrm{r}^{2}=0.4253\right.$, $\mathrm{p}=0.08$ ).

Table 3 Ankle-Brachial-Index for Control and Intervention Groups (Mean \pm SD)

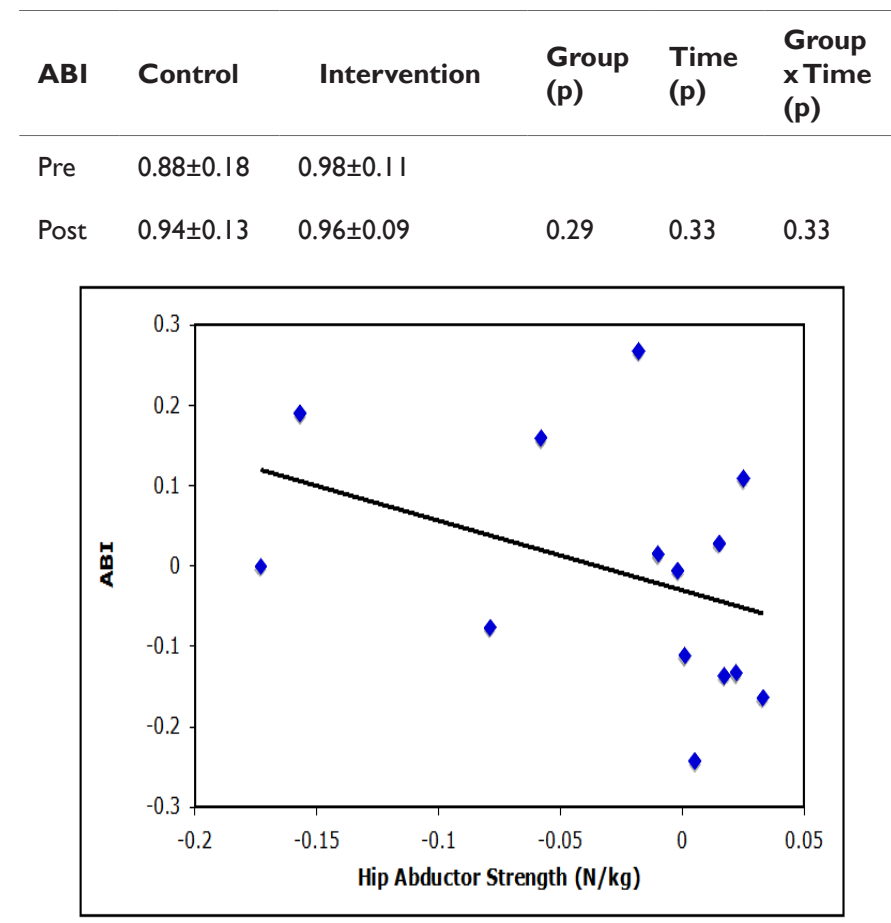

Figure I Change in Ankle-Brachial-Index $(\mathrm{mmHg} / \mathrm{mmHg})$ and Hip Abductor Strength $(\mathrm{N} / \mathrm{kg})$ for All Subjects.

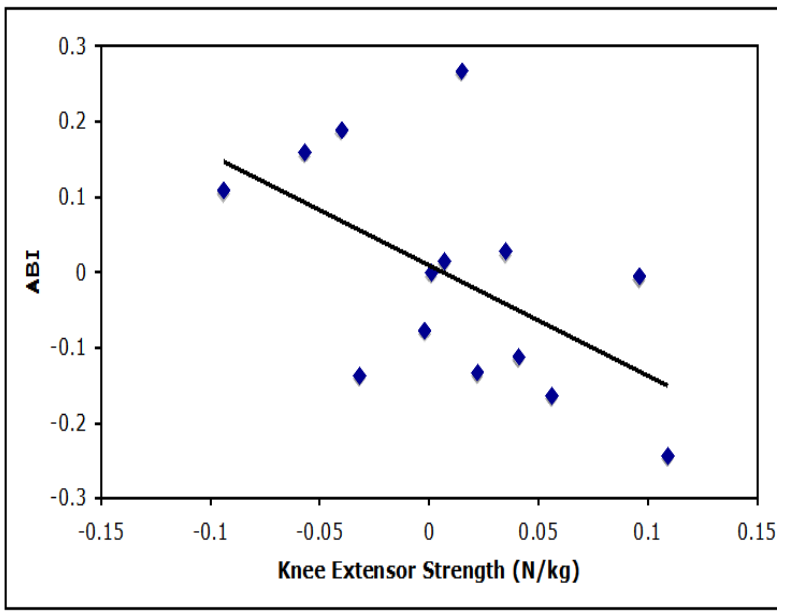

Figure 2 Change in Ankle-Brachial-Index $(\mathrm{mmHg} / \mathrm{mmHg})$ and Knee Extensor Strength $(\mathrm{N} / \mathrm{kg})$ for all subjects 
Table 4 r2 Values for Change in Muscular Strength and Ankle-Brachial-Index

\begin{tabular}{|c|c|c|c|c|c|c|c|}
\hline Group & $\begin{array}{l}\text { Plantar } \\
\text { flexion }\end{array}$ & Dorsiflexion & $\begin{array}{l}\text { Knee } \\
\text { extension }\end{array}$ & $\begin{array}{l}\text { Knee } \\
\text { flexion }\end{array}$ & $\begin{array}{l}\text { Hip } \\
\text { extension }\end{array}$ & Hip flexion & Hip abduction \\
\hline All & 0.021 & 0.075 & $0.306^{*}$ & 0.011 & 0.013 & 0.001 & $0.331 *$ \\
\hline Control & 0.055 & 0.262 & 0.403 & 0.187 & 0.24 & 0.017 & 0.003 \\
\hline Intervention & 0.002 & 0.006 & 0.425 & 0.094 & 0.033 & 0.07 & 0.417 \\
\hline
\end{tabular}

\section{Discussion}

The purpose of this current study was to investigate the effects of a 4 week resistance training program on muscular strength and anklebrachial-index in an OA population. We hypothesized that a resistancetraining program with this population would lead to an increase in muscular strength and improvement in ankle-brachial-index (ABI). We observed a trend towards significant improvement in hip abduction strength in the intervention group compared to the control group $(p=0.06)$. There were no significant training improvements in the remaining muscular strength or ABI measures. When assessing the relationship between the improvement in strength and $\mathrm{ABI}$ we observed trends towards a significant relationship in change in $\mathrm{ABI}$ and hip abduction strength and change in $\mathrm{ABI}$ and knee extension strength for the intervention group. The lack of significant findings may be due to the short duration of the resistance training period and/ or the low study sample size.

\section{Muscular strength measurements}

There were no significant differences between groups and across time with our muscular strength measurements. Previous studies have found significant muscular strength improvements in arthritic and non-arthritic populations. However, this resulted after six to twelve weeks of resistance training. ${ }^{11,13,14}$ Muscular strength values also differ in strength studies based on mode of testing, so it is difficult to compare values between previous studies and the current study. Manual muscle testing, used in our study, is not considered to be the gold standard for muscular strength testing; however in clinical practice with this population, it may be the most practical mode to determine force production without subjecting participants with joint pain to additional pain or discomfort.

A trend towards a significant interaction was observed in hip abductor strength in the intervention group. Studies have researched the impact of hip abductor weakness and fall risk. ${ }^{4,15}$ These studies investigated the impact of gait and obstacle challenges on maximal muscle utilization in older and younger adults. The vastuslateralis and gluteus medius of older adults were utilized close to 10 to $20 \%$ more to maximal capacity. The gluteus medius in this group was activated up to $46 \%$ on average during weight transfer obstacle training, while the younger group only came close to $23 \%$ on average. ${ }^{15}$ The current study demonstrated that a small increase in gluteus medius strength can occur in older adults with osteoarthritis in as little as four weeks. Some studies used functional exercises or isotonic exercises to increase strength that are commonly weak in older adults. ${ }^{7,13}$ Concentric functional activities like side-lying straight leg raises with ankle weights, along with additional pelvic stability exercises have been shown to increase muscular strength in this population. ${ }^{11,13}$ Functional exercises included in the current study were also similar to other exercises done in King et al.' ${ }^{11}$ study to improve gluteus medius strength, quadricep strength and dynamic balance.

\section{Ankle-brachial-index}

ABI did not exhibit a statistical difference between groups or across time. The literature does not fully support these findings. Research has shown that moderate changes to vasculature through resistance training, yet these training studies were longer than 4 weeks and performed in younger individuals or just males. ${ }^{14,16}$ Studies have shown that increased resting arterial diameter and enhanced resistance vessel function can be exhibited after resistance training in younger populations. ${ }^{16}$ This suggests that systolic blood pressure was lower after intervention, but cannot be compared to ABI because peripheral vasculature in the legs was not measured.

The current study used ABI to predict stenos is, if any, in individuals with OA, a population not yet assessed for stenos is in the lower extremities in the literature. The average pre ankle-brachialindex for the intervention group in the current study was $0.99 \pm 0.11$ (mean $\pm \mathrm{SD} ; \mathrm{mmHg} / \mathrm{mmHg}$ ) and decreased to $0.96 \pm 0.09$ (mmHg/ $\mathrm{mmHg}$ ). However, this decrease remained within the normal range $(0.90-1.50 \mathrm{mmHg} / \mathrm{mmHg})$. An ABI lower than $0.90 \mathrm{mmHg} / \mathrm{mmHg}$ is considered to be positive for some degree of stenosis; while, an ABI of $0.60 \mathrm{mmHg} / \mathrm{mmHg}$ or lower is considered diseased. ${ }^{17,18}$ McDermott et al. ${ }^{17}$ suggests that a value closer to $1.00 \mathrm{mmHg} / \mathrm{mmHg}$ is ideal. This would suggest that systolic blood pressure in the arms is the same as the lower legs. Our study exhibited that five out of the eight individuals in the intervention group improved his or her ABI by improving the values to be closer to $1.00 \mathrm{mmHg} / \mathrm{mmHg}$, implying more similar systolic arm and leg pressures. Vascular improvements after resistance training have not been typically quantified by measuring ABI improvements. The current study showed that while some participants improved ABI, the average ABI decreased. On average the intervention group increased in average systolic arm pressure. This could be due to events on the day of testing or other confounding variables that were hard to control for in this population. Overall, because this decrease in ABI did not drop below the normal range, we cannot suggest this alteration was clinically significant.

It should also be stated that the control group improved ABI. Vascular improvements are a factor that can result from cardiovascular training. ${ }^{719}$ Treadmill exercise in populations with peripheral arterial disease can improve endurance and walking ability, and show minor improvements $\left(1.53 \%\right.$ increase) in brachial artery flow dilation. ${ }^{19}$ Mode of cardiovascular training could have contributed to the increase in ankle-brachial-index. The mode in this study was determined by the degree of limitations as a result of osteoarthritis. Some studies have shown recumbent cycling as a mode to further increase muscle mass, muscle protein synthesis, and as a result increased blood flow to the lower extremities in older adults. ${ }^{7,19}$ These studies support our findings that exhibited increased $\mathrm{ABI}$ in the control group as a result of cardiovascular training.

\section{Relationships between change in strength and change in $\mathbf{A B I}$}

Significant relationships were observed for all participants for changes in hip abductor strength and ABI ( $\mathrm{p}=0.03)$, as well as knee extensor strength and ABI $(\mathrm{p}=0.04)$. For the intervention group, trendsof negative relationships were observed with hip abductor 
strength $(\mathrm{p}=0.08)$ and knee extensor strength $(\mathrm{p}=0.08)$ compared to ABI. Although not significant, this relationship could be classified as clinically significant. Both of these negative relationships displayed that as hip abductor strength or knee extensor strength increased, ABI decreased. The change in ABI was still with in the normal range $(0.90$ $-1.50 \mathrm{mmHg} / \mathrm{mmHg}$ ). If the ABI is higher than $0.90 \mathrm{mmHg} / \mathrm{mmHg}$ the risk for peripheral artery disease and stenos is in the lower limbs is significantly lower when compared to an $\mathrm{ABI}$ of less than 0.90 $\mathrm{mmHg} / \mathrm{mmHg}$. ${ }^{17,18}$

No studies have investigated the relationship between improvements in muscular strength and the respective changes in $\mathrm{ABI}$, especially with regards to OA patients. Decreased strength in knee extensors for older adults has been shown to hinder flexion and extension tasks, which can be detrimental in successfully clearing obstacles or engaging in functional tasks. ${ }^{4,21}$ It should be noted that we observed no significant differences in knee extensor strength and $\mathrm{ABI}$ in the control or intervention group. Resistance training studies have shown significant differences in older adults in these important muscle groups, but these numerous resistance training studies lasted longer than six weeks.

To our knowledge, this is the first study to investigate the relationship between strength and ABI in individuals with OA. Past studies have looked at vascular changes in older adults, but more commonly studied patients with cardiovascular disease or peripheral artery disease, not OA. Studies have shown increased fall risk for those with OA or peripheral artery disease, as well as in geriatric adults. ${ }^{4}, 15$ This could be a result of the disease state or a weakened musculoskeletal system. Strengthening of the muscles, particularly the hip abductors may reduce the risk of falls. ${ }^{20}$ The current study describes the relationship between changes in strength and $\mathrm{ABI}$ in $\mathrm{OA}$ populations after four weeks of resistance and/or cardio respiratory training, which has not been investigated thus far in the literature.

\section{Limitations}

Certain limitations to this study should be noted. A small sample size $(n=14)$ and the duration of the study ( 4 weeks) are the greatest limitations. Increasing the length of the study to eight weeks or more may result in statistical significance, especially in muscular strength. ${ }^{11,13,14,20}$ A power analysis (alpha level $=0.05$, power $=90 \%$ ) for hip abductor strength in the intervention group found that 28 subjects in each group would be needed to show statistical significance. The subject groups were predominately female, so sex may be considered a limitation. Transportation to the facility was another limitation to this study. Due to economic hardship and physical disability, some of the potential study participants had to rely on public transportation, which hurt recruitment and subject numbers.

\section{Future research}

Future research in the area of geriatric OA populations and resistance training is necessary. Based on the current literature, resistance training has been shown to benefit older adults with $\mathrm{OA}$ by decreasing pain, increasing strength and functionality, and impacting fall reduction. ${ }^{11,19,21} \mathrm{ABI}$ is a good inexpensive tool to field test vascular changes in a geriatric osteoarthritis population. More research is warranted to investigate at the relationship between ABI and muscular strength changes. The current study did not observe gait changes or measure changes in functional exercise tasks, which might be a good area for future research. Replication of this same study with a longer duration for the training period of eight to twelve weeks may show more significant changes muscular strength and ABI

\section{Conclusion}

The current study suggests that a four-week lower-body resistance training program with $\mathrm{OA}$ patients can elicit trends towards the improvement in hip abductor and knee extensor strength. Improvements in hip abductor and knee extensor strength resulted in clinically relevant alterations with ABI were observed as a result of resistance training. This type of lower body resistance training program appears to be beneficial for older adults with OA in an effort to reduce one's risk for peripheral artery disease. Therefore, older adults with OA should engage in a lower body resistance training program three days a week for at least four weeks to begin to observe improvements in lower body muscular strength and ankle-brachialindex.

\section{Acknowledgements}

None.

\section{Conflict of interest}

Authors declare there is no conflict of interest in publishing the article.

\section{References}

1. Felson DT, Zhang Y, Hannan MT, et al. The incidence and natural history of kneeosteoarthritis in the elderly. The framingham osteoarthritis study. Arthritis Rheum. 1985;38(10):1500-1505.

2. http://www.cdc.gov/arthritis/press/index.html

3. Der Ananian C, Wilcox S, Watkins K, et al. Factors associated with exercise participation in adults with arthritis. $J$ Aging Phys Act. 2008;16(2):125-143.

4. Hahn ME, Lee HJ, Chou LS. Increased muscular challenge i n older adults during obstructed gait. Gait Posture. 2005;22(4):356-361.

5. Miller A, Doll H, David J, et al. Impact of Musculoskeletal Disease on Quality of Life in Long-standing Acromegaly. European Journal of Endocrinology. 2008;158:587-593.

6. Arseven A, Guralnik JM, Kaleba E, et al. Does lower-extremity arterial disease predict future falling among older men and women. Angiology. 2008;58(6):725-733.

7. Topp R, Woolley S, Hornyak J , et al. The effect of dynamic versus isometric resistance training on pain and functioning among adults with osteoarthritis of the knee. Arch Phys Med Rehabil. 2002;83(9):1187-1195.

8. Lange SF, Trampisch HJ, Pittrow D, et al. Profound influence of different methods for determination of the ankle brachial index on the prevalence estimate of peripheral arterial disease. BMC Public Health. 2007;7:147-157.

9. Schroder F, Diehm N, Kareem S, et al. A modified calculation of ankle-brachial-index pressure index is far more sensitive in the detection of peripheral artery disease. J Vasc Surg. 2006;44(3):531-536.

10. Norkin CC, White DJ. Measurement of joint motion: A guide to goniometry, 3rd ed. PA. FA Davis Company, Philadelphia, USA; 2003. p. 261-275

11. King MB, Whipple RH, Gruman CA, et al. The performance enhancement project: Improving physical performance in older persons. Arch Phys Med Rehabil. 2002;83(8):1060-1069.

12. Whaley MH, Brubaker PH, Otto RM. ACSM's Guidelines for Exercise Testing and Prescription, 7th ed. k. L Williams \& Wilkins, New York, USA; 2006. p. 290-291. 
13. Ashe MC, Khan KM. Exercise prescription. J AmAcadOrthop Surg. 2004; $12: 21-27$.

14. Vad V, Hong HM, Zazzali M, et al. Exercise recommendations in athletes with early osteoarthritis of the knee. Sport Med. 2002;32(11):729-739.

15. Ades PA, Savage PD, Brochu M, et al. Resistance training increases total daily energy expenditure in disabled older women with coronary heart disease. J Appl Physiol. 2005;98(4):1280-1285.

16. Rakobowchuk M, McGowan CL, de Groot PC, et al. MacDonald MJ. Endothelial function of young healthy males following whole body resistance training. J Appl Physiol. 2005;98:2185-2190.

17. McDermott MM, Criqui MH, Greenland $\mathrm{P}$, et al. Leg strength in peripheral arterial disease: Associations with disease severity and lower-extremity performance. J Vasc Surg. 2004;39(3):523-530.
18. McDermott MM, Guralnik JM, AlbayM, et al. Impairments of muscles and nerves associated with peripheral arterial disease and their relationship with lower extremity functioning: The inCHIANTI study. $J \mathrm{Am}$ Geriatr Soc; 2004;52(3):405-410.

19. McDermott MM, Ades P, Guralnik J, et al. Treadmill exercise and resistance training in patients with peripheral artery disease with and without intermittent claudication. JAMA. 2009;301(2):165-174.

20. Chen HL, Lu TW, Wang TM, et al. Biomechanical strategies for successful obstacle crossing with the trailing limb in older adults with medial compartment knee osteoarthritis. J Biomech. 2008;41(4):753-761.

21. Hillard MJ, Martinez KM, Janssen I, et al. Lateral balance factors predict future falls in community-living older adults. Arch Phys Med Rehabil. 2008;89(9):1708-1713 